\title{
Narayana Prime Cordial Labeling of Complete Graph and Complete Bipartite Graph
}

\author{
S Venkatesh, B J Balamurugan
}

\begin{abstract}
Complete bipartite graph and complete graph are very important graphs and they find vital applications in the field of computer science. In this paper we compute the labels 0 and 1 to the edges of these graphs by satisfying the cordiality condition using prime numbers and Narayana numbers. We use divisibility concepts while computing the NP-cordial graphs.
\end{abstract}

Keywords: Narayana numbers, Prime numbers, NP cordial graph.

\section{INTRODUCTION}

A labeling of a graph $G(U, E)$ is an assignment of labels to the vertices in the vertex set $\mathrm{U}$ and the edges in the edge set $\mathrm{E}$ through mathematical functions [1]. In the year 1967, A. Rosa [9] introduced the idea of how to label the vertices of a graph. N.Lakshmi prasanna et.al, have listed some important applications of graph labeling in [5]. A survey, which is done by J.A.Gallian [3], gives day to day updation of graph labeling concepts. The basic definitions and terminologies used in this paper are referred to the text book written by Harary [4]. The study of Narayana numbers [6] forms part of the branch of mathematics called number theory. In combinatorial mathematics, the Narayana numbers occur in various counting problems. Since Narayana numbers have many applications, and graph labeling have often been motivated by practical problems, we have focused in Narayana labeling of graphs. In [7] Murali B.J et al have introduced the concept of Narayana prime cordial labeling. Subsequently in [2] it has been proved that the book graphs admit the Narayana prime cordial labeling. In this research article, we prove the existence of this Narayana prime cordial labeling to (i) complete bipartite graph $\left(K_{m, n}\right)$ and (ii) complete graph $\left(K_{n}\right)$.

\section{PRELIMINARIES}

The definitions pertaining to this paper are recalled in this section [8]

\section{A. Definition 2.1:}

Let $\mathbb{N}_{0}$ be the set of non-negative integers and let $k, n \in \mathbb{N}_{0}$

The Narayana numbers can be defined as

\footnotetext{
Revised Manuscript Received on December 16, 2019.

* Correspondence Author

S Venkatesh*, Mathematics Division, School of Advanced Sciences, VIT, Chennai, India. Email: venkatesh.2018@vitstudent.ac.in

B J Balamurugan, Mathematics Division, School of Advanced Sciences, VIT, Chennai, India. Email: balamurugan.bj@vit.ac.in
}

$$
\begin{aligned}
& N(n, k)=\frac{1}{n}\left(\begin{array}{l}
n \\
k
\end{array}\right)\left(\begin{array}{c}
n \\
k+1
\end{array}\right) ; 0 \leq k<n \\
& \text { Where }\left(\begin{array}{l}
n \\
k
\end{array}\right)=\frac{n !}{(n-k) ! k !}
\end{aligned}
$$

The Narayana numbers were first studied by Macmahon and later rediscovered by Narayana. The Narayana numbers are closely related to the Catalan numbers [10],

$$
C_{n}=\frac{1}{n+1}\left(\begin{array}{c}
2 n \\
n
\end{array}\right) \text { and } \sum_{k=0}^{n-1} N(n, k)=C_{n}
$$

Where $C_{n}$ is a Catalan number.

\section{B. Properties:}

(i) If $p$ is a prime number and $n=p^{m}$ for some $m \in \mathbb{N}_{0}$, then $p \mid N(n . k)$ for $1 \leq k \leq n-2$.

(ii) If $p$ is a prime number and $n=p^{m}-1$ for some $m \in \mathbb{N}_{0}$, then $p \nmid N(n, k)$ for $1 \leq k \leq n-1$.

\section{NP-CORDIAL LABELING OF GRAPHS}

The concept of Narayana prime cordial graphs introduced by Murali B.J et.al, in [7] has been discussed in this section, with an appropriate example.

\section{A. Definition 3.1: [7]}

Let $G(U, E)$ be a simple graph. A $1-1$ mapping $h: U \rightarrow \mathbb{N}_{0}$ is called a Narayana prime cordial labeling of the graph $G$ if there exists an edge mapping $h: E \rightarrow\{0,1\}$ such that

(i) For every $u v \in E$ and $u, v \in U$

$$
\begin{array}{r}
h^{*}(u v)=1 \text { if } p \mid N(h(u), h(v)) \text {, where } h(u)>h(v) \text { and } \\
h(u)=p^{m} \text { for some } m \in \mathbb{N}_{0} ; 1 \leq h(v) \leq \\
h(u)-2 \text { where pis a prime number } \\
=1 \text { if } p \mid N(h(v), h(u)) \text {, where } h(v)>h(u) \text { and } \\
h(v)=p^{m} \text { for some } m \in \mathbb{N}_{0} ; 1 \leq h(u) \leq \\
h(v)-2 \text { where pis a prime } n u m b e r \\
=0 \text { if } p \nmid N(h(u), h(v)) \text {, where } h(u)>h(v) \text { and }
\end{array}
$$




$$
\begin{array}{r}
h(u)=p^{m}-1 \text { for some } m \in \mathbb{N}_{0} ; 0 \leq h(v) \leq \\
h(u)-1 \text { where p is a prime number } \\
=0 \text { if } p \nmid N(h(v), h(u)), \text { where } h(v)>h(u) \text { and } \\
h(v)=p^{m}-1 \text { for some } m \in \mathbb{N}_{0} ; 0 \leq h(u) \leq \\
h(v)-1 \text { where pis a prime number }
\end{array}
$$

(ii) $\left|e_{h^{*}}(0)-e_{h^{*}}(1)\right| \leq 1$ where $e_{h^{*}}(0)$ and $e_{h^{*}}(1)$ represents respectively the number of edges with the label 0 and 1.

\section{B. Definition 3.2:}

A graph $G=(U, E)$ is called as a Narayana prime cordial graph if it admits a Narayana prime cordial labeling.

\section{Remark:}

In this paper we call, the Narayana prime cordial graph as "NP-cordial graph" for simplicity.

\section{Example 3.1:}

The graph given in the Fig. 1 is a NP-cordial graph.

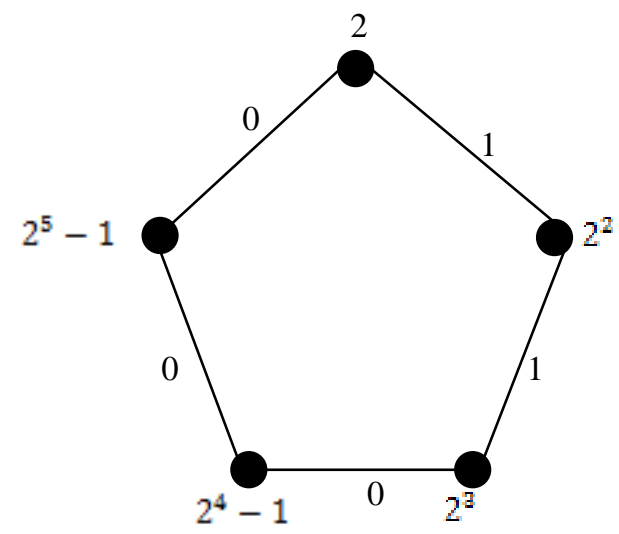

Fig. 1: A NP-cordial graph

\section{A. Theorem 1}

The complete bipartite graph $K_{m, n}$ admits a NP-cordial labeling for all positive integers $\operatorname{mand} n$.

Proof:

Let $K_{m, n}$ be the complete bipartite graph with vertex set $U=U_{1} \cup U_{2}$ such that where $U_{1}=\left\{u_{i} \mid 1 \leq i \leq m\right\}$ and $U_{2}=\left\{v_{j} \mid 1 \leq j \leq n\right\} \quad$ and $\quad$ edge $E=\left\{u_{i} v_{j} \mid u_{i} \in U_{1}, v_{j} \in U_{2} \forall i . j\right\}$. The graph $K_{m, n}$ has $m+n$ vertices and $m n$ edges.

Case (1) when $m \equiv 0(\bmod 2)$ and $n$ is any positive integer

Define a 1-1 mapping $h: U \rightarrow \mathbb{N}_{0}$ as

\section{NP-CORDIAL LABELING OF COMPLETE GRAPH AND COMPLETE BIPARTITE GRAPH}

$$
\begin{aligned}
& h\left(u_{i}\right)=\left\{\begin{array}{cc}
2^{n+i}, & 1 \leq i \leq m / 2 \\
2^{n+i}-1, & m / 2<i \leq m
\end{array}\right. \\
& h\left(v_{j}\right)=2^{j}, \quad 1 \leq j \leq n
\end{aligned}
$$

and $h^{*}: E \rightarrow\{0,1\}$ as in the definition 3.1

Through these functions, $\frac{n m}{2}$ edges get the number 0 and $\frac{n m}{2}$ edges get the number 1 .

Which means, $e_{h^{*}}(0)=n m / 2$ and $e_{h^{*}}(1)=n m / 2$ and $\left|e_{h^{*}}(0)-e_{h^{*}}(1)\right| \leq 1$ satisfied in this case.

Case (2) when $m$ is any positive integer and $n \equiv 0(\bmod 2)$

Define a 1-1 mapping $h: U \rightarrow \mathbb{N}_{0}$ as

$$
\begin{aligned}
& h\left(u_{i}\right)=2^{i}, 1 \leq i \leq m \\
& h\left(v_{j}\right)=\left\{\begin{array}{cc}
2^{m+j}, & 1 \leq j \leq \frac{n}{2} \\
2^{m+j}-1, & \frac{n}{2}<j \leq n
\end{array}\right.
\end{aligned}
$$

and $h^{*}: E \rightarrow\{0,1\}$ as in the definition 3.1

Through these functions, $\frac{n m}{2}$ edges get the number 0 and $\frac{n m}{2}$ edges get the number 1 .

Which means, $e_{h^{*}}(0)=n m / 2$ and $e_{h^{*}}(1)=n m / 2$ and $\left|e_{h^{*}}(0)-e_{h^{*}}(1)\right| \leq 1$ satisfied in this case.

Case (3) when $m \equiv 1(\bmod 2)$ and $n \equiv 1(\bmod 2)$ and $n=m$

Define a 1-1 mapping $h: U \rightarrow \mathbb{N}_{0}$ as

$$
\begin{aligned}
& h\left(u_{i}\right)=\left\{\begin{array}{cc}
2^{i}, \quad 1 \leq i<m \\
2^{2 n}-1, \quad i=m
\end{array}\right. \\
& h\left(v_{j}\right)=\left\{\begin{array}{cc}
2^{n+j-1}, \quad 1 \leq j \leq \frac{n+1}{2} \\
2^{n+j-1}-1, \quad \frac{n+1}{2}<j \leq n
\end{array}\right.
\end{aligned}
$$

and $h^{*}: E \rightarrow\{0,1\}$ as in the definition 3.1

Through these functions, $\frac{n m+1}{2}$ edges get the number 0 and $\frac{n m-1}{2}$ edges get the number 1 .

Which means, $e_{h^{*}}(0)=\frac{n m+1}{2}$ and $e_{h^{*}}(1)=\frac{n m-1}{2}$ and $\quad\left|e_{h^{*}}(0)-e_{h^{*}}(1)\right| \leq 1$ satisfied in this case.

Case 
$m \equiv 1(\bmod 2), n \equiv 1(\bmod 2)$ and $m>n$

Define a 1-1 mapping $h: U \rightarrow \mathbb{N}_{0}$ as

$$
\begin{aligned}
& h\left(u_{i}\right)=\left\{\begin{array}{c}
2^{i}, \quad 1 \leq i \leq \frac{m-1}{2} \\
2^{i+1}, \quad \frac{m-1}{2}<i \leq m
\end{array}\right. \\
& h\left(v_{j}\right)=\left\{\begin{array}{c}
2^{m+j+1}, \quad 1 \leq j \leq \frac{n-1}{2} \\
2^{m / 2}-1, \quad j=\frac{n+1}{2} \\
2^{m+j}-1, \quad \frac{n+1}{2}<j \leq n
\end{array}\right.
\end{aligned}
$$

and $h^{*}: E \rightarrow\{0,1\}$ as in the definition 3.1

Through these functions, $\frac{n m+1}{2}$ edges get the number 1 and $\frac{n m-1}{2}$ edges gets the number 0 .

$$
\text { Which means, } \quad e_{h^{*}}(1)=\frac{n m+1}{2} \quad \text { and }
$$$$
e_{h^{*}}(0)=\frac{n m-1}{2} \text { and }\left|e_{h^{*}}(0)-e_{h^{*}}(1)\right| \leq 1 \text { satisfied in this }
$$
case.

Case (5) when $m \equiv 1(\bmod 2), n \equiv 1(\bmod 2)$ and $m<n$

Define a 1-1 mapping $h: U \rightarrow \mathbb{N}_{0}$ as

$$
h\left(v_{j}\right)=\left\{\begin{array}{cc}
2^{j}, & 1 \leq j \leq \frac{n-1}{2} \\
2^{j+1}, & \frac{n-1}{2}<j \leq n
\end{array}\right.
$$

and $h^{*}: E \rightarrow\{0,1\}$ as in the definition 3.1

Through these functions, $\frac{n m+1}{2}$ edges get the number 1 and $\frac{n m-1}{2}$ edges gets the number 0 .

Which means, $\quad e_{h^{*}}(1)=\frac{n m+1}{2} \quad$ and $e_{h^{*}}(0)=\frac{n m-1}{2}$ and $\left|e_{h^{*}}(0)-e_{h^{*}}(1)\right| \leq 1$ satisfied in this case.

In all cases the condition $\left|e_{h^{*}}(0)-e_{h^{*}}(1)\right| \leq 1$ is satisfied and therefore the complete bipartite graph $K_{m, n}$ admits a NP-cordial labeling.

The following examples illustrate the NP-cordial labeling of the complete bipartite graph $K_{m, n}$.

\section{B. Example 4.1:}

When $m=4, n=5$ the NP-cordial labeling of $K_{4,5}$ is given in Fig. 2

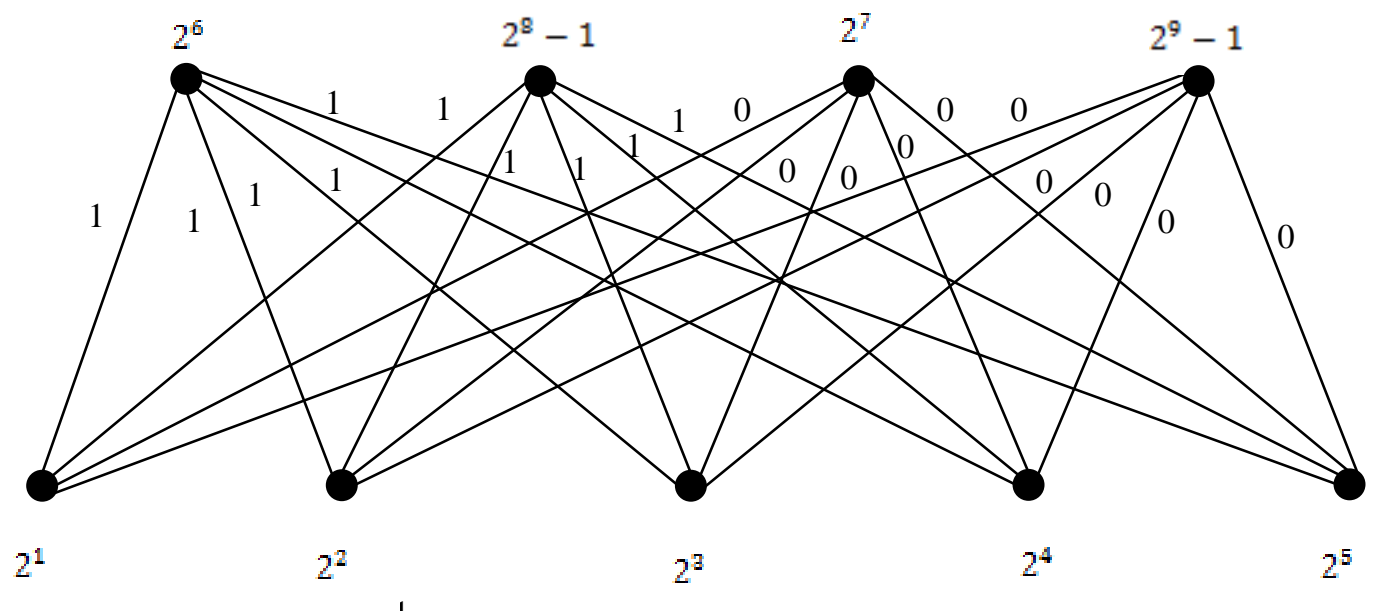

$$
h\left(u_{i}\right)=\left\{\begin{array}{cc}
2^{n+i+1}, & 2^{2} \\
2^{n / 2}-1, & i=\frac{m+1}{2} \\
2^{n+i}-1, & \frac{m+1}{2}<i \leq m
\end{array}\right.
$$




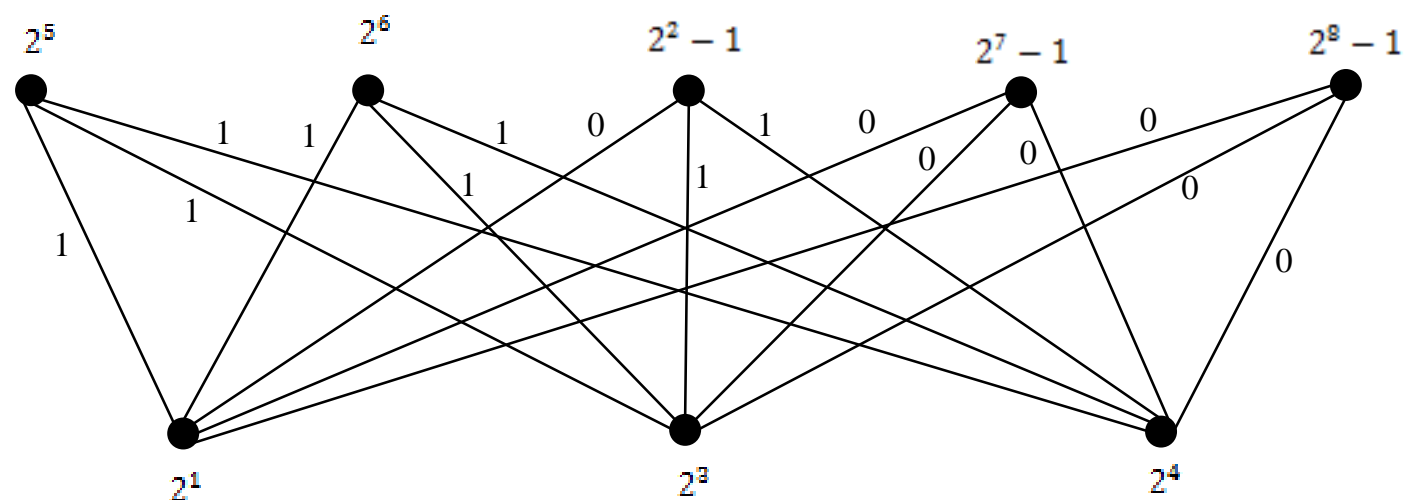

Fig. 3: NP-cordial labeling of $K_{5,3}$

\section{Theorem 2:}

The complete graph $K_{n}$ for all positive $n$ admits a NP-cordial labeling.

Proof

Let $K_{n}$ be the complete graph with $\mathrm{n}$ vertices.

Let $U=\left\{v_{i} \mid 1 \leq i \leq n\right\} \quad$ be the vertex set and $E=\left\{v_{i} v_{j} \mid v_{i}, v_{j} \in U \forall i, j\right\}$ be the edge set of $K_{n}$. The graph $K_{n}$ has $n$ vertices and $\frac{n(n-1)}{2}$ edges.

Case (1): $n \equiv 5,7(\bmod 8)$

Define a 1-1 mapping $h: U \rightarrow \mathbb{N}_{0}$ as

$$
h\left(v_{i}\right)=\left\{\begin{aligned}
2^{i+1}, & i=1,3,5, \ldots, n-2 \text { and } i=\left\lceil\frac{3 n}{4}\right\rceil \\
2^{i+1}-1, & \text { otherwise }
\end{aligned}\right.
$$

and $h^{*}: E \rightarrow\{0,1\}$ as in the definition 3.1

Subcase (1a) when $n \equiv 5(\bmod 8)$

In this case $K_{n}$ have even number of edges.

Through these functions, $\frac{n(n-1)}{4}$ edges get the number 0 and $\frac{n(n-1)}{4}$ edges gets the number 1 .

Which means, $e_{h^{*}}(0)=\frac{n(n-1)}{4}$ and $e_{h^{*}}(1)=\frac{n(n-1)}{4}$ and $\left|e_{h^{*}}(0)-e_{h^{*}}(1)\right| \leq 1$ satisfied in this case.

Subcase (1b) when $n \equiv 7(\bmod 8)$

In this case $K_{n}$ have odd number of edges.
Through these functions, $\frac{n(n-1)-2}{4}$ edges get the number 0 and $\frac{n(n-1)+2}{4}$ edges gets the number 1 .

Which means, $\quad e_{h^{*}}(0)=\frac{n(n-1)-2}{4} \quad$ and $e_{h^{*}}(1)=\frac{n(n-1)+2}{4}$ and $\left|e_{h^{*}}(0)-e_{h^{*}}(1)\right| \leq 1$ satisfied in this case.

Case (2): $n \equiv 1,3(\bmod 8)$

Define a 1-1 mapping $h: U \rightarrow \mathbb{N}_{0}$ as

$$
h\left(v_{i}\right)=\left\{\begin{array}{cc}
2^{i+1}, \quad i=1,3,5, \ldots, n-2 \quad \text { and } i=2, i=\left\lfloor\frac{3 n}{4}\right\rfloor \\
2^{i+1}-1, \quad \text { otherwise }
\end{array}\right.
$$

and $h^{*}: E \rightarrow\{0,1\}$ as in the definition 3.1

Subcase (2a) when $n \equiv 1(\bmod 8)$

In this case $K_{n}$ have even number of edges.

Through these functions, $\frac{n(n-1)}{4}$ edges get the number 0 and $\frac{n(n-1)}{4}$ edges get the number 1 .

Which means, $\quad e_{h^{*}}(0)=\frac{n(n-1)}{4} \quad$ and $e_{h^{*}}(1)=\frac{n(n-1)}{4}$ and $\left|e_{h^{*}}(0)-e_{h^{*}}(1)\right| \leq 1$ satisfied in this case.

Subcase (2b) when $n \equiv 3(\bmod 8)$

In this case $K_{n}$ have odd number of edges

Through these functions, $\frac{n(n-1)-2}{4}$ edges get the number 0 and $\frac{n(n-1)+2}{4}$

edges gets the number 1 . 
Which means, $\quad e_{h^{*}}(0)=\frac{n(n-1)-2}{4} \quad$ and $e_{h^{*}}(1)=\frac{n(n-1)+2}{4}$ and $\left|e_{h^{*}}(0)-e_{h^{*}}(1)\right| \leq 1$ satisfied in this case.

Case (3) $n \equiv 2,4(\bmod 8)$

Define a 1-1 mapping $h: U \rightarrow \mathbb{N}_{0}$ as

$$
h\left(v_{i}\right)=\left\{\begin{aligned}
2^{i+1}, & i=1,3,5, \ldots, n-1 \quad \text { and } i=\frac{n}{4}+1 \\
2^{i+1}-1, & \text { otherwise }
\end{aligned}\right.
$$

and $h^{*}: E \rightarrow\{0,1\}$ as in the definition 3.1

Subcase (3a) when $n \equiv 4(\bmod 8)$

In this case $K_{n}$ have even number of edges.

Through these functions, $\frac{n(n-1)}{4}$ edges get the number 0 and $\frac{n(n-1)}{4}$ edges gets the number 1 .

Which means, $e_{h^{*}}(0)=\frac{n(n-1)}{4}$ and $e_{h^{*}}(1)=\frac{n(n-1)}{4}$ and $\left|e_{h^{*}}(0)-e_{h^{*}}(1)\right| \leq 1$ satisfied in this case.

Subcase (3b) when $n \equiv 2(\bmod 8)$

In this case $K_{n}$ have odd number of edges.

Through these functions, $\frac{n(n-1)-2}{4}$ edges get the number 0 and $\frac{n(n-1)+2}{4}$ edges gets the number 1 .

Which means, $\quad e_{h^{*}}(0)=\frac{n(n-1)-2}{4}$ and

$e_{h^{*}}(1)=\frac{n(n-1)+2}{4}$ and $\left|e_{h^{*}}(0)-e_{h^{*}}(1)\right| \leq 1$ satisfied in this case.

Case (4) $n \equiv 0,6(\bmod 8)$ and $n \neq 8$

Define a 1-1 mapping $h: U \rightarrow \mathbb{N}_{0}$ as

$$
h\left(v_{i}\right)=\left\{\begin{aligned}
2^{i+1}, i=1,3,5, \ldots, n-1 \quad \text { and } i=2, i=\frac{n}{4} \\
2^{i+1}-1, \text { otherwise }
\end{aligned}\right.
$$

and $h^{*}: E \rightarrow\{0,1\}$ as in the definition 3.1

Subcase (4a) when $n \equiv 0(\bmod 8)$ and $n \neq 8$

In this case $K_{n}$ have even number of edges.

Through these functions, $\frac{n(n-1)}{4}$ edges get the number 0 and $\frac{n(n-1)}{4}$ edges gets the number 1 .
Which means, $e_{h^{*}}(0)=\frac{n(n-1)}{4}$ and $e_{h^{*}}(1)=\frac{n(n-1)}{4}$ and $\left|e_{h^{*}}(0)-e_{h^{*}}(1)\right| \leq 1$ satisfied in this case.

Subcase (4b) when $n \equiv 6(\bmod 8)$ and $n \neq 6$

In this case $K_{n}$ have odd number of edges.

Through these functions, $\frac{n(n-1)-2}{4}$ edges get the number 0 and $\frac{n(n-1)+2}{4}$ edges get the number 1 .

Which means, $\quad e_{h^{*}}(0)=\frac{n(n-1)-2}{4} \quad$ and $e_{h^{*}}(1)=\frac{n(n-1)+2}{4}$ and $\left|e_{h^{*}}(0)-e_{h^{*}}(1)\right| \leq 1$ satisfied in this case.

Subcase (4c) when $n=6$

Through these functions 8 edges get the number 0 and 7 edges get the number 1 .

Which means, $e_{h^{*}}(0)=8$ and $e_{h^{*}}(1)=7$ and $\left|e_{h^{*}}(0)-e_{h^{*}}(1)\right| \leq 1$ satisfied in this case.

Case (5) $n=8$

Define a 1-1 mapping $h: U \rightarrow \mathbb{N}_{0}$ as

$$
\begin{aligned}
& h\left(v_{1}\right)=2, h\left(v_{2}\right)=2^{3}, h\left(v_{3}\right)=2^{4}-1, h\left(v_{4}\right)=2^{5}, \\
& h\left(v_{5}\right)=2^{6}, h\left(v_{6}\right)=2^{7}-1, h\left(v_{7}\right)=2^{8}, h\left(v_{8}\right)=2^{9}-1
\end{aligned}
$$
and $h^{*}: E \rightarrow\{0,1\}$ as in the definition 3.1

Through these functions, 14 edges get the number 0 and 14 edges gets the number 1 .

Which means, $e_{h^{*}}(0)=14$ and $e_{h^{*}}(1)=14$ and $\left|e_{h^{*}}(0)-e_{h^{*}}(1)\right| \leq 1$ satisfied in this case.

In all cases the condition $\left|e_{h^{*}}(0)-e_{h^{*}}(1)\right| \leq 1$ was satisfied therefore complete graph admits a NP-cordial labeling.

The following examples illustrate the NP-cordial labeling of the complete graph $K_{n}$.

\section{E. Example 4.3:}

The NP-cordial labeling of $K_{5}$ is given in the Fig. 4 


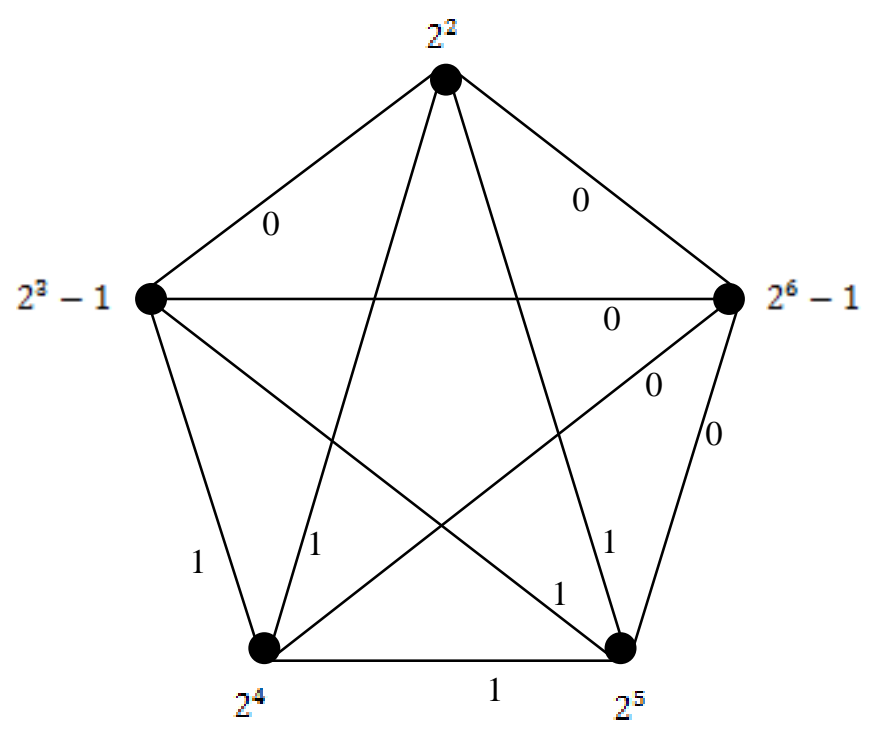

Fig. 4: NP-cordial labeling of $K_{5}$

\section{F. Example 4.4:}

The NP-cordial labeling of $K_{6}$ is given in the Fig. 5

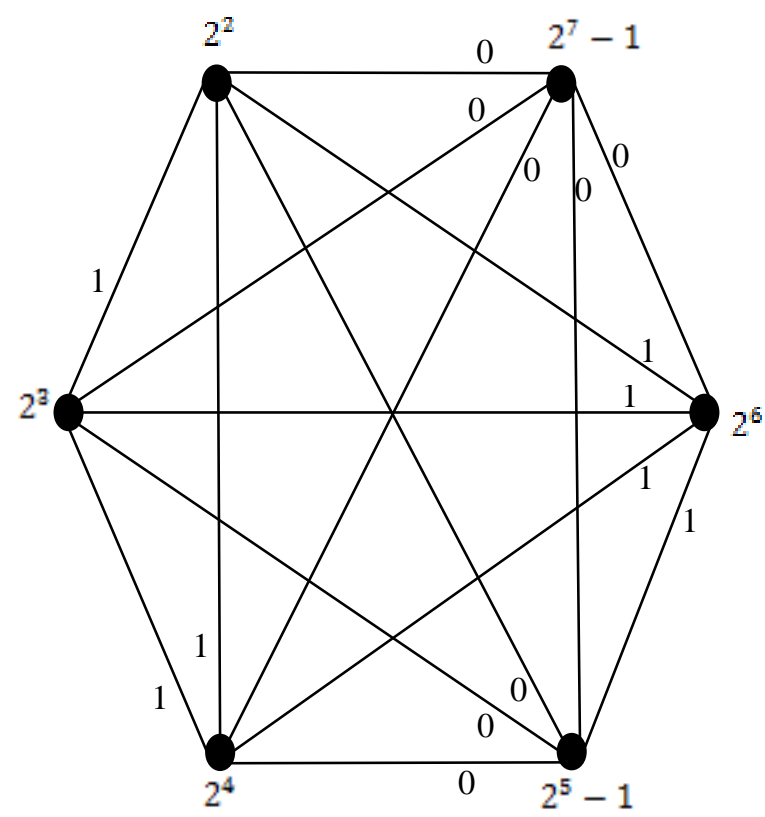

Fig. 5: NP-cordial labeling of $K_{6}$

\section{CONCLUSION}

The NP-cordial labeling of two interesting graphs viz. (i) Complete bipartite graph and (ii) Complete graph have been computed in this article. Computing NP-cordial labeling of other family of graphs is a potential area of research in graph theory and future scope for the readers.

\section{REFERENCES}

1. B.D. Acharya, S.M. Hegde, Arithmetic Graphs, J. Graph Theory, 14(3) (1990), 275-299.

2. B.J. Balamurugan, K. Thirusangu, B.J. Murali, J.Venkateswara Rao, J.(2019), Computation of Narayana Prime Cordial Labeling of Book Graphs, Applied Mathematics and Scientific Computing.

3. J.A. Gallian, A Dynamic Survey of Graph Labeling, Electronic Journal of Combinatorics, DS6 (2018).
4. F. Harary, Graph Theory, Addison-Wesley, Reading Mass (1972).

5. N. Lakshmi Prasana, K. Saravanthi, Nagalla Sudhakar, Applications of Graph Labeling in Major Areas of Computer Science, International Journal of Research in Computer and Communication Technology, 3(8) (2014).

6. Miklos Bona, Bruce E. Sagan, On Divisibility of Narayana Numbers by Primes, Journal of Integer Sequences, 8 (2005).

7. B.J. Murali, K. Thirusangu, B.J. Balamurugan, Narayana Prime Cordial Labeling of Graphs, International Journal of Pure and Applied Mathematics. 117(13), (2017) 1-8.

8. M. Randic, D. Morales, O. Arauji, Higher-order Lucas Numbers, Divulgaciones Mathematicas, 16(2) (2008), 275-283.

9. A. Rosa, On Certain Valuations of the Vertices of a Graph, In Theory of Graphs (Internat. Sympos. Rome. 1966), Gordan and Breach. Newyork. Dunod. Paris (1967), 349-359.

10. Thomas Koshy, Catalan Numbers with Applications, Oxford University Press (2009).

\section{AUTHORS PROFILE}

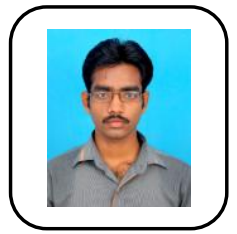

S Venkatesh received his M.Sc. and M.Phil. degrees in Mathematics from the University of Madras, Chennai, India. He has one year of experience at Undergraduate and Postgraduate level courses. He cleared CSIR-NET (JRF). He is currently pursuing $\mathrm{Ph} . \mathrm{D}$. in Mathematics. His area of research is Graph Theory.

Dr.B.J.Balamurugan received his $\mathrm{Ph} . \mathrm{D}$. degree in Mathematics from the

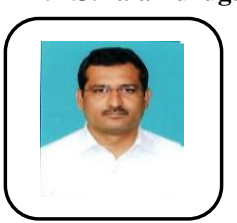
University of Madras, Chennai, India. Currently, he is an Assistant Professor (Senior) of Mathematics in the School of Advanced Sciences at VIT University, Chennai Campus, Chennai, India. He has more than 22 years of teaching experience at Undergraduate and Postgraduate level courses. Dr.B.J.Balamurugan has published more than 32 research papers in various journals and conference proceedings. His research interest includes graph theory, graph grammars, fuzzy logic and Petri nets. 